\title{
Interstitial lung disease in a rheumatic electrician
}

\section{Case history}

The case presented here concerns a male, born in 1931, who was an electrician for 34 years. He had frequent and close contact with asbestos, chrysotile and amphiboles in large buildings in which he fitted electric cables and worked in ventilation shafts. In addition, the patient smoked for 48 pack-years. His health problems began in 1982 with severe rheumatoid arthritis, and he retired in 1987 at the age of 56 years because of this disease.

The patient first presented in 1989, in the rheumatology clinic, and showed typical signs of joint and bone deformities. He had already been treated with steroids and anti-inflammatory agents. He had several subcutaneous nodules in his wrists and elbows, and a very strongly positive rheumatoid biology, with an elevated erythrocyte sedimentation rate and an elevated capillary osmotic pressure.

In 1989, treatment with prednisolone (5-10 mg daily) and methotrexate (7.5-17.5 mg weekly) was initiated. However, bouts of arthritis and tenosynovitis still recurred, even with subsequent adaptations of treatment doses and surgical interventions on the hands and wrists.

\section{Case referral}

The patient was referred to pneumologists in 1994, because of the presence of cough, breathlessness and a small volume of sputum in the morning. No wheezing was found on physical examination, but bilateral crackles were clearly identified at both lung bases. The lung function test results were forced vital capacity and total lung capacity (TLC) within normal limits, with mild obstruction, which improved after bronchodilatation.

Figure 1 shows a chest radiograph from 1994, where some irregular markings in the mid-zones could be identified, along with a blunting of the costofrenic angle. The computed tomography (CT) scans obtained at this time are shown in figure 2. CT scans demonstrated interstitial lung disease, essentially subpleural, ground-glass, linear opacities and nodules. It was also clear that the patient had been exposed to asbestos, due to the presence of pleural plaques, calcified on the diaphragm and in both posterior parts of the thorax.

The patient underwent a bronchoalveolar lavage (BAL), which showed the following: cytology 360,000 cells. $\mathrm{mL}^{-1} ; 46 \%$ lymphocytes (T4/T8); 45\% macrophages; 3\% neutrophils; and $6 \%$ eosinophils. A mineral analysis was performed and 5.4 asbestos bodies. $\mathrm{mL}^{-1}$ were found. All cultures were negative for bacteria, microbacteria, moulds and viruses, and no neoplastic cells were detected.

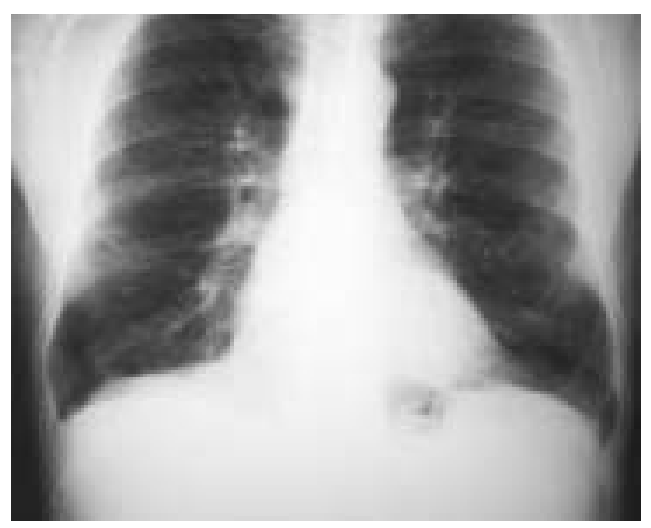

Figure 1

Chest radiography taken in 1994.

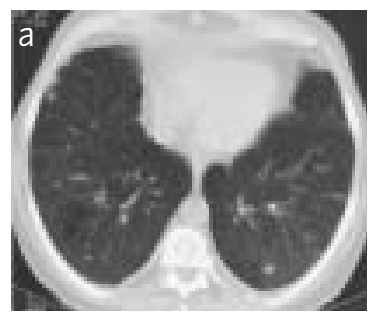

Figure 2

CT scans taken in 1994.
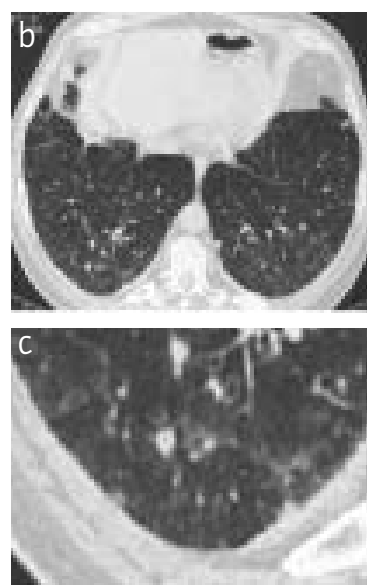

\section{P. De Vuyst}

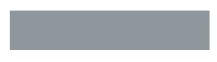

Erasme Hospital

Route de Lennik 808

1070 Brussels

Belgium

Fax: 3225554411

E-mail:pdevuyst@ulb.ac.be

\section{Task 1}

At this time, what would you consider to be the most likely diagnosis?

\section{Asbestosis.}

2. Pulmonary manifestation of rheumatoid arthritis. 3. An association of both.

\section{Methotrexate toxicity. \\ 5. Idiopathic pulmonary fibrosis (IPF).}

Task 2

Do you consider that in this case asbestos exposure was sufficient to induce asbestosis?

\section{Yes.}

2. No.

3. Don't know. 


\section{Answer 1 \\ The wrong answer in this case was IPF, as the CT appearances were not pathognomonic of IPF.}

\section{Answer 2}

At this time, the answer should be "I don't know". More information is needed.

\section{Asbestos exposure}

The duration (34 years) and latency (41 years) of asbestos exposure of the patient were compatible with asbestos-related disease. It is known that asbestos exposure is frequent among electricians, although the cumulative exposure is only usually enough to cause pleural plaques or mesothelioma, but too low to result in fibrosis of the lung parenchyma.

In order to obtain an objective assessment of cumulative exposure in the patient, two processes were used, as follows.

1) Retrospective evaluation by industrial engineers using a job/exposure matrix established in France. Using this technique, it was calculated that cumulative exposure was 85 fibres per $\mathrm{mL}$ of breathed air per year of exposure. If you accept the recent publications on asbestosis, which have been reviewed by Mossman and CHURG [1], asbestosis is not expected to occur below a minimum threshold dose in the range 25-100 fibres per $\mathrm{mL}$ per year, depending on the type of fibre. In addition, another study [2], concerning a retired workforce from an asbestos transformation factory, performed the same evaluation of cumulative exposure (table 1). It can be seen from this analysis that the present patient would be placed in the category with $\sim 6 \%$ of lesions of fibrosis on high-resolution CT.

2) Mineral analysis of BAL. Using this technique, $>5$ asbestos bodies per $\mathrm{mL}$ were found, and the accepted reference thresholds for exposure to asbestos [3] state that the general population usually has $<1$ asbestos bodies per $\mathrm{mL}$ fluid.
Table 1 Lung fibrosis in a retired workforce from an asbestos transformation factory

\begin{tabular}{|lcccc|}
\hline & \multicolumn{4}{c|}{ Cumulative exposure fibres per $m$ L per year } \\
& $<25$ & $25-99$ & $100-199$ & $\geq 200$ \\
HRCT: fibrosis & $0 \%$ & $5.8 \%$ & $5.1 \%$ & $12.9 \%$ \\
Chest radiography: $\geq 1 / 0$ & $18 \%$ & $18.7 \%$ & $14.6 \%$ & $16.1 \%$ \\
\hline
\end{tabular}

Figure 3 shows the results of a BAL fluid mineral analysis over $\sim 20$ years [4], which shows that patients with asbestosis usually have very high asbestos body counts, with a median count of 100; however, some cases lie in the category of retention of the current patient, $\sim 5$ asbestosis bodies per $\mathrm{mL}$, although this is the type of retention usually related to pleural plaques and mesothelioma. Therefore, asbestosis is possible in this patient, but with a limited cumulative dose.

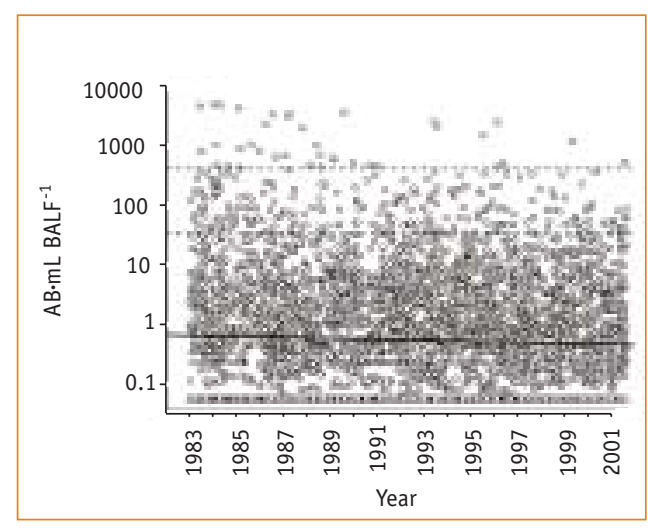

Figure 3

$B A L$ fluid mineral analysis. Modified with permission from [5].

\section{Medical conclusions}

At this time, the probable diagnosis was thought to be interstitial lung disease associated with rheumatoid arthritis, based on the severity of the disease, the $C T$ aspects with presence of nodules and ground-glass opacities, and the BAL results showing lymphosytosis. The patient had pleural plaques, and co-existing asbestosis was not excluded. He also had mild chronic obstructive lung disease (COPD) due to smoking. In addition, methotrexate toxicity was ruled out, as the patient had been taking methotrexate for 5 years.

Task 3

If you were the medical expert in this case, would you accept the diagnosis of asbestosis?

1. Yes.

2. No. 


\section{Answer 3}

As this case was in Belgium, the Central Occupational Disease Fund accepted the diagnosis of asbestosis, since both asbestos and connective tissue disorders could have contributed to lung fibrosis, and the patient subsequently received compensation (30\% disability).

Lung biopsy was not performed at this time, and, as there was relative confidence in the diagnosis of rheumatoid lung disease, the patient was treated for that. There is no treatment for asbestosis, so surgical biopsy was not performed.

Rheumatoid lung disease was further treated using steroids and methotrexate. He completely stopped smoking in 1986, $\beta_{2}$-agonists were prescribed for his COPD with partly reversible obstruction, and a CT follow-up was performed.

Functionally, over about 10 years, the patient's condition did not alter. On CT evaluation, a surprising finding was that the patient's nodules seemed to have disappeared, although the linear opacities were still present. Pleural plaques did increase and became more calcified.

\section{Final diagnosis}

In 2002, the patient experienced a worsening of cough, and, because of this, signs of cancer were looked for. Cancer was detected in the left upper lobe with involvement of the mediastinum and the pulmonary artery. The patient could not be surgically operated upon at that time, because of the mediastinal involvement and, in addition, bone metastases. The patient did not respond to chemotherapy. He got an infection in his lung and, hence, a salvage pneumectomy was performed in 2002. He subsequently broke his leg and died in July 2002 from massive pneumonia.

Figure 4 shows a chest radiograph just before pneumectomy. Radiography showed the lung cancer excavated with infection. Macroscopically (figure 5), subpleural linear fibrosis could be detected. Histologically (figure 6), the visceral pleura appeared quite thickened, with subpleural fibrosis, patchy fibrosis and some inflammatory infiltrates. In addition, in some places, there was evidence of subepithelial fibroblastic foci, which are suggestive of usual interstitial pneumonia (UIP). Intra-alveolar organisation, as seen in bronchiolitis obliterans organising pneumonia (BOOP), was observed, as well as bronchiolar fibroblasty. Some areas were reminiscent of fibrotic non-specific interstitial pneumonia (NSIP) with homogenous fibrosis. And finally, some cicatricial rheumatoid nodules with a thick fibrotic wall were detected.

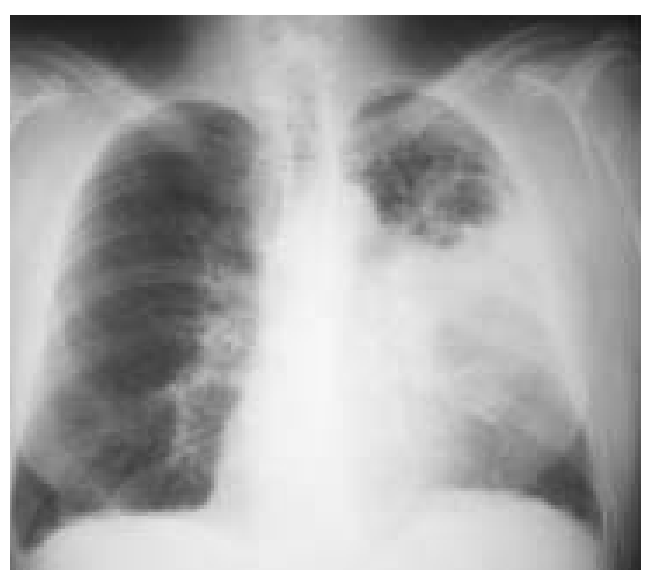

Figure 4

Chest radiography taken in 2002.

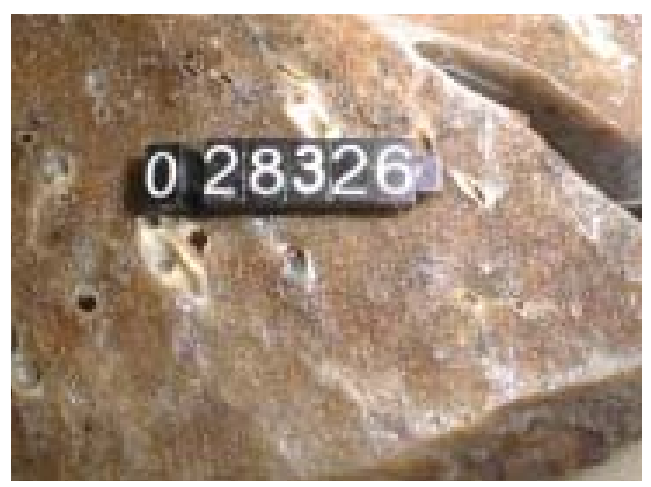

Figure 5

Macroscopic findings in 2002.
This case presentation was taken from a Grand Round, held at the ERS Congress, Glasgow 2004.

Go to www.ersnet.org/elearning for further information. 

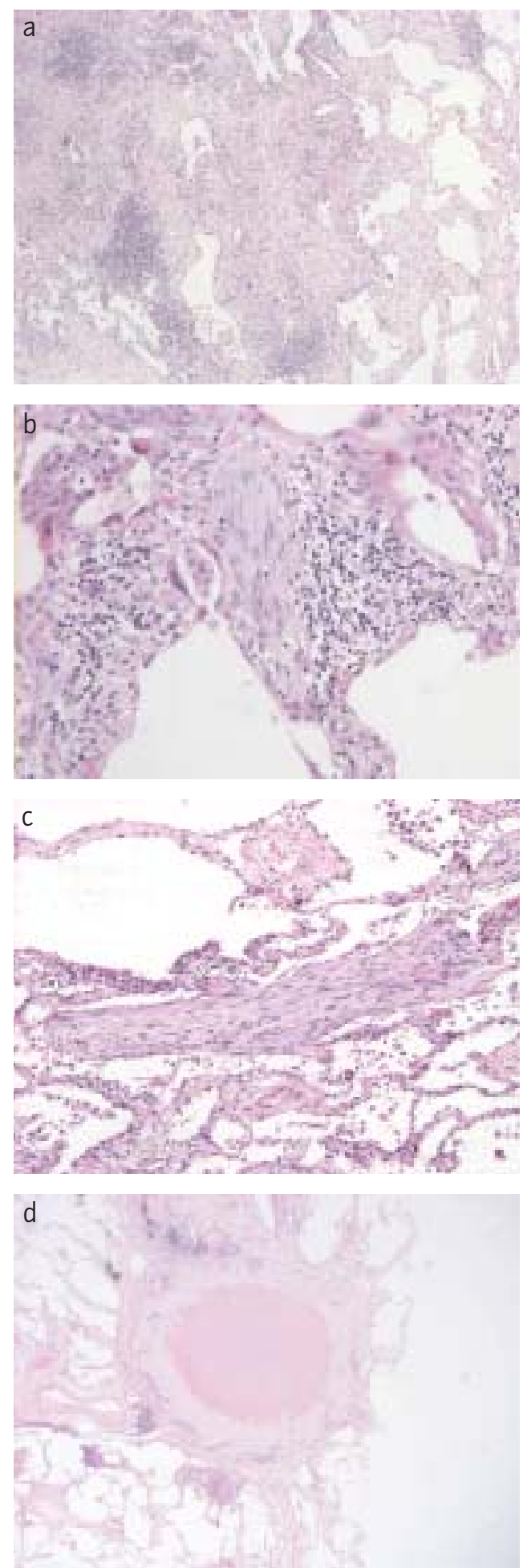

Figure 6

Histological findings in 2002: a) subpleural patchy fibrosis, b) fibroblastic foci, c) BOOP-like lesion and d) rheumatoid nodule.
In summary, the diagnosis was complicated and a second opinion was sought. Prof. Nicholson (Brompton Hospital, London, UK) concluded the following: interstitial fibrosis with mixed appearances of fibrotic NSIP, UIP and areas of BOOP, compatible with connective tissue disorders-associated interstitial lung disease. He also confirmed that the necrotic nodules were cicatricial rheumatoid nodules and he found very occasional asbestos bodies in sections. Importantly, he also stated that asbestos bodies were: "Below the amount expected for the degree of interstitial fibrosis" [4].

Mineral analysis was performed with optical microscopy, which showed 15,496 asbestos bodies per gram of dry lung, and with electron microscopy, which showed 4.5 million fibres per gram dry lung (among which 2.2 million were $<5$ $\mu \mathrm{m}$ in length). The types of fibre found included: crocidolite $76 \%$, amosite $22 \%$ and chrysotile $2 \%$.

The medical conclusion this time was adenocarcinoma of left upper lobe with retro-obstructive pneumonia, rheumatoid lung (interstitial fibrosis and cicatricial rheumatoid nodules), pleural plaques and mild COPD.

\section{Task 4 \\ At this stage, if you were the medical expert for this case, would you accept the diagnosis of occupa- tional lung cancer? \\ 1. Yes. \\ 2. No.}




\begin{abstract}
Answer 4
The diagnosis of occupational lung cancer was accepted again by the Central Occupational Disease Fund Belgium. The reason for this was that the patient's fibrosis had been accepted as asbestosis and, therefore, automatically, his cancer was accepted as a complication of asbestosis. In addition, using objective measures, the patient had a high cumulative exposure of $>25$ fibres per $\mathrm{mL}$ per year.
\end{abstract}

\section{Task 5}

What do you consider as possible causal factors for the pleural plaques in this patient?

1. Asbestos exposure.

2. Tobacco smoking.

3. Asbestos and smoking.

4. Neither asbestos nor smoking.

\section{Task 6}

What do you consider as possible causal factors for lung cancer in this patient?

1. Asbestos exposure.

2. Tobacco smoking.

3. Asbestos and smoking.

4. Neither asbestos nor smoking.

\section{Task 7}

What do you consider as possible causal factor for rheumatoid arthritis and, consequently, rheumatoid lung in this patient?

1. Asbestos exposure.

2. Tobacco smoking.

3. Asbestos and smoking.

4. Neither asbestos nor smoking.
Answer 5

Asbestos and smoking.

Answer 6

Asbestos and smoking.

Answer 7

Neither asbestos nor smoking.

\section{Discussion}

There is no convincing evidence of an association between asbestosis or asbestos exposure and rheumatoid arthritis. On the contrary, there is a considerable amount of evidence that rheumatoid arthritis can be associated with silicosis and silica exposure, which is termed Caplan's syndrome, and has been documented in granite workers, coal and gold miners, and dental technicians.

Cigarette smoking has been increasingly shown in longitudinal and case-controlled studies to be an important risk factor for both the incidence and severity of rheumatoid arthritis, especially in seropositive males $[6,7]$. The only causal factor for bilateral calcified pleural plaques is exposure to asbestos or other fibrous minerals, never smoking. This cannot be seen in any other condition, except with exposure to other fibres, such as Zeolite in Turkey.

Therefore, discussions about the toxicity of tobacco are not complete, and it is possible that many diseases other than lung disease can be caused by tobacco.

\section{References}

1. Mossman BT, Churg A. Mechanisms in the pathogenesis of asbestosis and silicosis. Am J Respir Crit Care Med 1998; 157: 1666-1680.

2. Paris C, Letourneux M, Catilina P, Frimat P, Sobaszek A, Boutin C. [Prevention programs for people exposed to asbestos: results of the ongoing activities]. Rev Mal Respir 1999; 16: 1332-1349.

3. De Vuyst $P$, Karjalainen $A$, Dumortier $P$, et al. Guidelines for mineral fibre analyses in biological samples: report of the ERS Working Group. European Respiratory Society. Eur Respir J 1998; 11: 1416--1426.

4. Tansey $D$, Wells $A U$, Colby TV, et al. Variations in histological patterns of interstitial pneumonia between connective tissue disorders and their relationship to prognosis. Histopathology 2004; 44: 585-596.

5. Albano SA, Santana-Sahagun E, Weisman MH. Cigarette smoking and rheumatoid arthritis. Semin Arthritis Rheum 2001; 31: 146-159.

6. Hutchinson D, Shepstone L, Moots R, Lear JT, Lynch MP. Heavy cigarette smoking is strongly associated with rheumatoid arthritis (RA), particularly in patients without a family history of RA. Ann Rheum Dis 2001; 60: 223-227. 